\title{
Evaluación del efecto genotóxico en pacientes con malaria de una región de Colombia
}

\author{
Diana P. Cuesta, Lina M. Martínez, Mónica M. Massaro, Camilo A. Agudelo, Isabel C. Hurtado, \\ Diana C. Miranda, Ana I. Garrido, Johan S. Lopera Verónica A. Arango e Isabel C. Ortiz
}

\section{Evaluation of the genotoxic effect of malaria in a cohort of patients in Medellín and Quibdó}

Introduction: During malaria infection, both parasite and host are under the effects of oxidative stress due to the increased production of reactive oxygen species, which can induce DNA damage by its genotoxic effects. Objective: To evaluate genotoxic effects in human lymphocytes in a cohort of patients with malaria from Medellin and Quibdó. Methods: We performed an observational cross sectional study in 100 individuals with malaria and 100 healthy controls. Patients infected with Plasmodium consulting the Institute Colombiano of Medicina Tropical of Medellin and the Hospital Ismael Roldán Valencia of Quibdó were included. Genotoxic effects (genetic damage) was analysed by electrophoresis using alkaline single cell gel (Commet assay). Results: The average of tail length of malaria samples $(26.9 \pm 9.8)$ was significantly higher than of controls $(14.8 \pm 3.2)(p<0.01)$. Conclusion: In our study population, malaria infection was associated with increased genotoxicity, while other variables such as smoking, antimalarial treatment, and occupation were not.

Key words: Comet assay; reactive oxygen species; oxidative stress; genotoxicity; Plasmodium.

Palabras clave: Ensayo cometa, especies reactivas de oxígeno, estrés oxidativo, genotoxicidad, Plasmodium.

\section{Introducción}

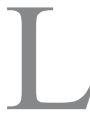

a malaria es una enfermedad infecciosa causada por protozoos del género Plasmodium, que afecta a más de 200 millones de personas, de las cuales 1-2 millones mueren cada año. En Colombia, $85 \%$ del territorio nacional está propenso a la transmisión de la malaria, con áreas endémicas en el Bajo Cauca, Orinoco, Pacífico y Amazonas. Las especies del género Plasmodium prevalentes en el país son: $P$. vivax, que causa 54 a $60 \%$ de los casos, $P$. falciparum, de 30 a $45 \%$ de los casos y $P$. malariae menos de $1 \%$. En la región del Pacífico se produce un fenómeno diferente ya que allí $P$. falciparum causa $75 \%$ de los casos ${ }^{1}$. De acuerdo con las condiciones geográficas, $85 \%$ del territorio está por debajo de los $1.800 \mathrm{msnm}$, en los cuales cerca de $65 \%$ de la población está en riesgo de sufrir malaria. En promedio hay 130.000 a 150.000 casos de malaria cada año, lo que demuestra un incremento en el número de casos en el último año. Según la Organización Panamericana de la Salud, en Colombia durante el año 2002, ocurrieron 195.719 casos de malaria entre 22.403 millones de personas expuestas, un incremento de $22,5 \%$ de casos respecto al año anterior ${ }^{1}$. En 2006, Antioquia reportó 41.012 casos de malaria por $P$. falciparum, $P$. vivax y mixto, para una tasa media de 716 casos/100 mil habitantes ${ }^{2}$.

Durante la infección de la malaria, tanto el parásito como el hospedero están bajo los efectos de estrés oxidativo, dado que se aumenta la producción de especies reactivas del oxígeno (ERO) (anión superóxido, peróxido de hidrógeno, radicales hidroxilo y ácido hipocloroso) $)^{3,4}$. El aumento de radicales libres en el organismo tiene como finalidad servir como mecanismo de tratamiento sobre los eritrocitos parasitados para inhibir la infección y destruir el parásito. Sin embargo, las ERO se ven envueltas en cambios patológicos sobre todas las células de los tejidos, donde el estrés oxidativo al que se está sometido no es selectivo. Los mecanismos específicos a través de los cuales la infección por Plasmodium genera ERO son desconocidos. Sin embargo, se ha evidenciado la generación de estrés oxidativo a través del desequilibrio entre la producción de ERO y la reducción de vías antioxidantes. Una importante aproximación se ha considerado a través de la actividad fagocítica que se inicia en las parasitemias por Plasmodium sumado a la producción de citokinas, metabolismo de la membrana eritrocitaria e inclusive el uso de medicamentos antimaláricos ${ }^{4}$.

En diversas publicaciones se ha encontrado que las ERO pueden inducir daños en el ADN. Ray y cols., reportaron que el óxido nítrico (NO) y el malondialdehido (MDA) tenían un rol importante en el daño al ADN, produciendo intercambio de cromátidas hermanas (ICH) en cáncer de mama y que existía una correlación directa entre estos niveles y la malignidad del tumor. También
Universidad Pontificia

Bolivariana, Medellín, Colombia. Escuela de Ciencias de la Salud Facultad de Medicina (ICH, DCM, AIG), Grupo de Biología de Sistemas, (LMM, CAA, JSL, VAA, ICO), Grupo de Salud Pública (DPC).

Universidad de Antioquia, Medellín, Colombia. Facultad Nacional de Salud Pública (DPC)

Instituto Neurológico de Colombia, Medellín, Colombia. Departamento de Investigación y Docencia (MMM).

Establecimientos donde se realizó el trabajo: Hospital Ismael Roldán Valencia, Quibdó (Chocó); y EI Instituto de Medicina Tropical, Medellín (Antioquia).

Este trabajo estuvo financiado por el Centro de Investigación para el Desarrollo y la Innovación CIDI.

Los autores declaran no tener conflicto de intereses.

Recibido: 25 de septiembre de 2013

Aceptado: 12 de junio de 2014

Correspondencia a: Isabel Cristina Ortiz Trujillo Isabel.ortiz@upb.edu.co 
observaron la expresión de otros radicales libres como el anión superóxido, que ayudan a perpetuar el daño genético, demostrando así el gran poder genotóxico que tienen los radicales libres ${ }^{1,5-7}$.

Una de las asociaciones más significativas entre la infección por Plasmodium y neoplasias se encuentra en el linfoma de Burkitt, uno de los linfomas no Hodgkin monoclonal de células B cuya prevalencia es bastante alta en países de África ecuatorial, coincidiendo en regiones endémicas para malaria. Varios estudios han demostrado que la parasitemia, la prevalencia de infección por Plasmodium y algunas variantes genéticas se relacionan directamente con el desarrollo del linforma. Si bien los mecanismos biológicos y oncogénicos no se han establecido completamente, se ha identificado la activación atípica de células $\mathrm{B}$ de memoria cuya inducción puede estar mediada por el imbalance oxidativo propio de la infección parasitaria ${ }^{8}$.

En Colombia son pocos los estudios sobre daño genotóxico, estrés oxidativo y enfermedades infecciosas a pesar de su alta prevalencia. Esta investigación se propuso evaluar el efecto genotóxico (daño genético, medido como la longitud de cola en $\mu \mathrm{m}$ ) en linfocitos humanos de pacientes con malaria, con el fin de aportar al conocimiento de la fisiopatología de la malaria y las consecuencias derivadas de la infección por Plasmodium.

\section{Material y Método}

\section{Tipo de estudio}

Se realizó un estudio cross sectional analítico en personas con malaria y controles sanos con valoración transversal del efecto (daño genético).

\section{Población de estudio}

Los pacientes corresponden a población del área de influencia de la ESE Hospital Ismael Roldán Valencia, institución de baja complejidad responsable de las necesidades en el primer nivel de atención de la población de la ciudad de Quibdó, región del Pacífico colombiano. Se incluyeron además, pacientes que consultaron en el Instituto de Medicina Tropical de Medellín, Colombia. Los controles sanos fueron pareados por edad y sexo en relación a la población afectada. Corresponden a población de los programas de promoción y prevención de la misma institución, sin antecedentes epidemiológicos, clínicos o de laboratorio de malaria. Al momento de la toma de muestra ninguno de los controles sanos tenía sospecha de infección por Plasmodium sp.

\section{Muestra}

Se determinó un tamaño de muestra por conveniencia de 200 personas: 100 individuos con malaria y 100 controles sanos. Todos los participantes firmaron un consentimiento informado para su inclusión en el estudio y se contó con la aprobación del Comité de Ética de Investigación de la Universidad Pontificia Bolivariana.

Se identificaron como casos aquellos pacientes con criterios clínicos, epidemiológicos y de laboratorio, según la guía clínica de la Organización Panamericana de la Salud. En los criterios clínicos se consideró la presencia de episodios febriles, paroxismos de fiebre y escalofríos, cefalea, síntomas gastrointestinales, mialgias, artralgias, náuseas, vómito y presencia de esplenomegalia. Los criterios epidemiológicos considerados fueron los antecedentes de exposición definidos como la permanencia en áreas con transmisión activa de enfermedad en los últimos 15 días a la toma de la muestra, nexo epidemiológico (tiempo y lugar) con personas que hubieran padecido malaria y antecedentes de medicación antimalárica en las últimas cuatro semanas. Con relación a las pruebas de laboratorio, la gota gruesa es el método recomendado como primera opción diagnóstica. La prueba fue realizada por personal entrenado en la técnica con curvas significativas de aprendizaje. No se realizó diagnóstico a través de extendido de sangre periférica ni prueba rápida. Se consideró negativa la gota gruesa en aquellas muestras cuya lectura en al menos 200 campos microscópicos no evidenció presencia de formas parasitarias. En aquellos casos en los que predominó la sospecha clínica y el nexo epidemiológico y la muestra fue negativa, se repitió la prueba a las $24 \mathrm{~h}^{9}$.

\section{Evaluación del daño en el ADN}

Para la evaluación del daño, se tomó una muestra de 5 $\mathrm{ml}$ de sangre periférica heparinizada $(50 \mathrm{U} / \mathrm{mol}$ de heparina sódica), mantenida en hielo seco para su conservación y transporte al laboratorio de Proteómica de la Facultad de Medicina de la Universidad Pontificia Bolivariana, donde fue procesada y analizada por un investigador capacitado previa estandarización de las técnicas de procesamiento de muestras. La sangre se centrifugó a 1.200 rpm por 7 min, se descartó el sobrenadante y se resuspendió el sedimento en solución salina balanceada con tampón fosfato (PBS) en proporción 1:1. Posteriormente, se aislaron los linfocitos utilizando el método de separación por densidades con $3 \mathrm{ml}$ de Hystopaque-1077 (Sigma St. Louis, Missouri). La suspensión se centrifugó a 2.000 rpm por 30 min, se realizaron dos lavados con PBS y finalmente, se resuspendió el sedimento en $1 \mathrm{ml}$ de PBS y se determinó su viabilidad a través del ensayo de exclusión con el colorante vital azul de Tripano $(0,4 \%)^{10}$, así:

$\%$ viabilidad $=\left(\mathrm{n}\right.$ de céls. vivas $/ \mathrm{N}^{\circ}$ de céls. totales $) * 100$

La genotoxicidad (definida como daño en el ADN) se detectó a través del ensayo Cometa alcalino siguiendo el protocolo propuesto por Singh y cols. ${ }^{11}$, modificado 
por Pandrangi y cols. ${ }^{12}$. Se utilizaron concentraciones de $5 \times 10^{4}$ céls $/ \mathrm{ml}$ suspendidas en agarosa de bajo punto de fusión, LMA (low melting agarose) $(0.5 \%$ en PBS libre de $\mathrm{Ca}^{++}, \mathrm{Mg}^{++}$) en placas pre-tratadas con $100 \mathrm{ml} \mathrm{de}$ agarosa de punto de fusión normal NMA (normal melting agarose) $\left(0.5 \%\right.$ en PBS libre de $\left.\mathrm{Ca}^{++}, \mathrm{Mg}^{++}\right)$. Se colocaron las laminillas y después de seis minutos a $4^{\circ} \mathrm{C}$ se retiraron, se agregó una tercera capa de LMA $(0,5 \%$ en PBS libre de $\left.\mathrm{Ca}^{++}, \mathrm{Mg}^{++}\right)$y se llevaron a $4^{\circ} \mathrm{C}$ por seis minutos; se removieron las laminillas y las placas se colocaron en solución de lisis recién preparada $(2,5 \mathrm{M} \mathrm{NaCl}, 1 \%$ de sarcosinato de sodio, 0.1M EDTA, 10mM Tris- $\mathrm{HCl} 10 \%$ dimetil sulfóxido (DMSO) 1\% de Tritón X 100) pH 10, por $60 \mathrm{~min}$ a $4^{\circ} \mathrm{C}$. Después de la lisis, los portaobjetos se lavaron con PBS libre de $\mathrm{Ca}^{2++} \mathrm{y} \mathrm{Mg}^{2++}$ y se colocaron en una cámara de electroforesis horizontal con una solución tampón de electroforesis (0,3 M NaOH, $1 \mathrm{mM}$ EDTA, $\mathrm{pH}$ =13) (1 a $2 \mathrm{~mL}$ por encima de las placas) durante $20 \mathrm{~min}$ en un cuarto oscuro a $4^{\circ} \mathrm{C}$, para la desnaturalización del ADN y para optimizar las rupturas de los sitios apurínicos (AP) antes del corrido electroforético, el cual se realizó durante $30 \mathrm{~min}$ a $25 \mathrm{~V}$ y $300 \mathrm{~mA}$. Luego, las placas se lavaron por $10 \mathrm{~min}$ con solución neutralizante $(0,4 \mathrm{M}$ Tris$\mathrm{HCl} ; \mathrm{pH}=7,5)$ y se deshidrataron con metanol absoluto.

Posteriormente, las placas se colorearon con bromuro de etidio $(0,2 \%)$ y se visualizaron con un aumento de $40 \mathrm{X}$ en un microscopio de fluorescencia con un filtro de excitación de 515-560 nm. Para cada individuo se analizaron 50 células por placa (total dos placas) y se determinó la longitud $(\mu \mathrm{m})$ de la cola de los cometas. Como control positivo se utilizó peróxido de hidrógeno $\left(\mathrm{H}_{2} \mathrm{O}_{2}\right)(100$ $\mu \mathrm{g} / \mathrm{ml}$ ). Los parámetros validados para determinar daño evaluado a través de este ensayo son: migración del ADN, concentración de ADN en la cola, momento de cola, momento Olive, entre otros ${ }^{13}$. En este estudio se utilizó como parámetro de medida la longitud de cola $(\mu \mathrm{m})^{14-17}$.

\section{Análisis estadístico}

Se describieron las características sociodemográficas y de la infección con frecuencias y porcentajes, para evaluar las diferencias de los promedios de la longitud de cola entre pacientes con malaria e individuos sanos (controles), se utilizó la prueba t de Student con varianzas diferentes. Se evaluó el efecto del grupo (100 pacientes con malaria y 100 individuos sanos) en conjunto con sexo, ocupación (ama de casa, estudiante, agricultor, minero, oficios varios) y hábito de fumar (sí/no) sobre los promedios de la longitud de cola $(\mu \mathrm{m})$ con el test Análisis de Varianza (ANOVA) bifactorial. En el grupo de pacientes con malaria se compararon los promedios de la longitud de cola $(\mu \mathrm{m})$ entre especies de Plasmodium (falciparum y vivax), la infección (primoinfección/reinfección) y el tratamiento (sí/no) mediante la prueba t de Student. Se consideró un nivel de significancia $\alpha=0,05$. La normalidad en la dis- tribución de los datos se determinó a través de las pruebas Kolmogorov-Smirnov y Shapiro-Wilk. Para comparar la parasitemia con el promedio de la longitud de cola se utilizó el coeficiente de correlación de Spearman. En todos los casos se utilizó el paquete estadístico: Statistica 7.0 (StatSoft, Inc. Tulsa, OK, USA).

\section{Resultados}

Cincuenta y cinco por ciento de los pacientes con diagnóstico de malaria fueron hombres. Más de $70 \%$ de los pacientes residía en Quibdó. Las ocupaciones principales de las personas analizadas fueron: ama de casa y otros oficios que incluyen oficinista, recepcionista, estadístico, entre otros. El antecedente de tabaquismo se encontró en 13\% (Tabla 1). La primoinfección se observó en 59\% de los pacientes y las especies predominantes en el grupo fueron $P$. falciparum $(77 \%)$ y $P$. vivax $(20 \%)$. Treinta y tres pacientes de los reinfectados estaban recibiendo tratamiento con cloroquina (Tabla 2).

\begin{tabular}{|llc|}
\hline \multicolumn{2}{|c|}{$\begin{array}{c}\text { Tabla 1. Características sociodemográficas de los } \\
\text { participantes del estudio }\end{array}$} \\
\hline Característica & & $\mathbf{n}$ \\
Procedencia & Quibdó & 73 \\
& Medellín & 27 \\
Sexo & Masculino & 55 \\
Tabaquismo actual & Femenino & 45 \\
& Sí & 13 \\
Ocupación & No & 87 \\
& Ama de casa & 21 \\
& Otros & 27 \\
& Canteras & 11 \\
& Estudiantes & 11 \\
& Oficios varios & 9 \\
& Jornaleros & 8 \\
& Desempleados & 13 \\
\hline
\end{tabular}

$\begin{aligned} & \text { Tabla 2. Características de la infección de los pacientes } \\
& \text { con malaria }\end{aligned}$
\begin{tabular}{llc} 
Característica & $\mathbf{n}=100$ \\
\multirow{4}{*}{ Tipo de infección } & Primoinfección & $\%$ \\
& Reinfección & 59 \\
Tipo de Plasmodium & falciparum & 41 \\
& vivax & 77 \\
Tratamiento & Mixto & 20 \\
& Sí & 3 \\
& No & 33 \\
& & 67
\end{tabular}




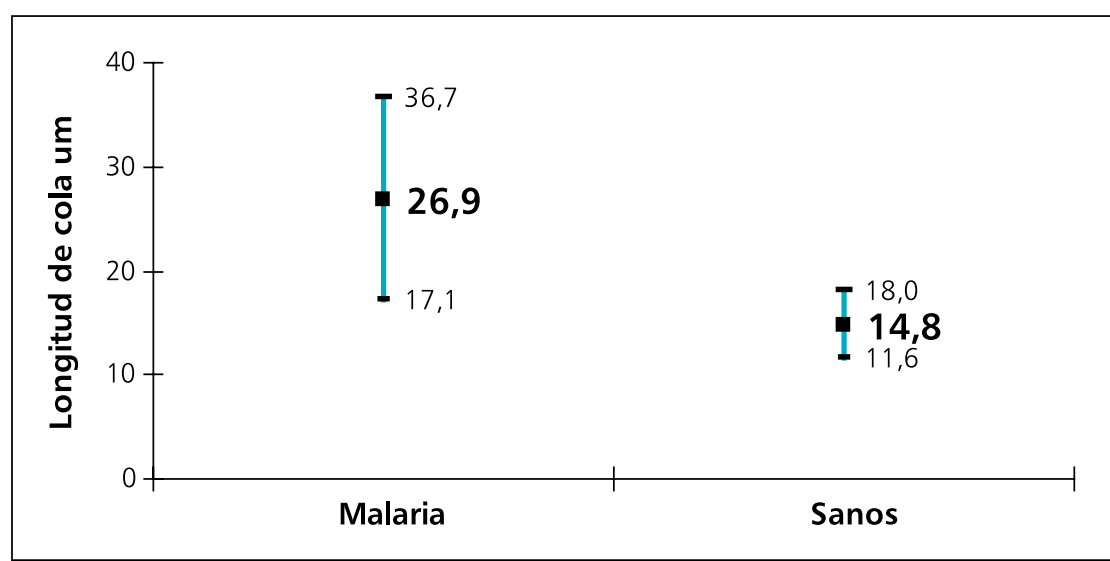

Figura 1. Promedio de la longitud de cola $(\mu \mathrm{m})$ y desviación estándar de pacientes con malaria e individuos sanos $(p<0,001)$. generó daño genotóxico evidenciado a través del ensayo Cometa. Es probable que el daño hubiera sido producido por las especies reactivas del oxígeno cuyo origen es variado; tanto el parásito como la respuesta inmune del hospedero, son responsables de su producción ${ }^{4}$. Sin embargo, se hace necesario comprobar la asociación entre la producción de ERO secundario a la infección por malaria y el tipo de daño en el ADN encontrado en este estudio.

Las enfermedades infecciosas generan ERO, que producen daños (quiebres de cadena sencilla y de cadena doble) en el ADN, que pueden ser detectados a través de diferentes metodologías, como la electroforesis en gel de

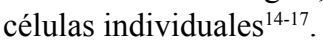

Es característico en la parasitemia por Plasmodium la actividad muy dinámica de las enzimas oxidantes y antioxidantes $^{18-20}$. En esta infección se disminuyen las enzimas con función anti-oxidante (catalasas, superóxido dismutasas, glutation peroxidasas) pudiendo generar especies reactivas del oxígeno y que los fármacos antimaláricos (cloroquina y primaquina) aumentan la producción de ERO en linfocitos y macrófagos para la destrucción del microorganismo ${ }^{1}$. Sanni y cols., en $1993^{21}$ y Pabón y cols., en el $2003^{3}$, llegaron a la conclusión que en la infección por Plasmodium están aumentadas las enzimas antioxidantes, lo cual demuestra de una manera indirecta, la producción de ERO durante la malaria. El aumento de estas sustancias en pacientes con malaria, podría evidenciar el daño genético encontrado en comparación con individuos sanos. Dado que en el estudio no hubo medición sérica de marcadores de estrés oxidativo, se realizó una aproximación a la valoración de la genotoxicidad por la infección parasitaria a través de pruebas de asociación entre la longitud de cola de los casos y la parasitemia y no se encontró una significancia estadística que evidenciara un efecto proporcional y directo entre ambas variables.

Nuestros hallazgos coinciden con lo reportado por Weitherg, en $1990^{22}$, quien concluyó que algunas especies reactivas del oxígeno inducen daño en el ADN en células de mamíferos cultivadas. En 1992, Eisenberg y cols., ${ }^{23}$ demostraron que el oxígeno singlete (especie reactiva del oxígeno que es producida gracias a la presión atmosférica) puede generar intercambio de cromátidas hermanas, en células de hígado humano. Ray y cols., en el año $2001^{5}$, encontraron que el óxido nítrico (NO) y el malondialdehido (MDA) juegan un papel importante en el daño del material genético a través del aumento en la expresión de anión superóxido, que ayudan a perpetuar el daño genético, demostrando así el gran poder genotóxico que tienen los radicales libres.

Finalmente, las variables sociodemográficas, la parasitemia y el tratamiento no se asociaron a daño genético identificado a través del ensayo Cometa. Algunas de estas variables pudieron generar daño, no a través de la producción de ERO y no detectable por la técnica utilizada, 
aunque muchos autores han demostrado asociación entre genotoxicidad y factores extrínsecos como la exposición laboral, la contaminación ambiental y el tabaquismo entre otros $^{24-27}$. Para controlar posibles variables confusoras como las anteriores, la muestra fue pareada y se realizó el análisis estadístico buscando la influencia de cada una de ellas en el daño genético encontrado y no se encontró relación estadísticamente significativa (Tabla 3 ).

\section{Limitaciones}

Existen diversas técnicas para evaluar genotoxicidad como la inducción de micronúcleos, Ensayo Cometa, el intercambio de cromátides hermanas y el test de alteraciones cromosómicas. Ninguna de ellas es completamente eficaz para identificar el daño genotóxico dados los diversos mecanismos de daño y reparación celular. En este estudio la técnica escogida fue el Ensayo Cometa Alcalino, técnica introducida en 1988. Una de las limitaciones de la técnica es la no detección de aneunógenos y la posibilidad de identificar daños que han sido ya reparados. Sin embargo, siendo una técnica con más de 20 años de implementación, su utilización como evaluador de daño en el material genético ha crecido exponencialmente en aplicabilidad y es tema central de discusión por expertos. En el año 2013, durante el X International Comet Assay Workshop $^{28}$ se consideraron las nuevas aplicaciones de esta técnica en enfermedades humanas como respuesta en inflamación, daño oxidativo en diabetes mellitus y enfermedades metabólicas hereditarias.

Otra limitación del estudio fue la correlación entre el efecto genotóxico y su relación con el tiempo de aclaramiento de la parasitemia. Esta relación no se puede establecer en un estudio transversal.

Los resultados obtenidos en este estudio evidencian el daño genotóxico en pacientes con malaria pero en ausencia de medición de marcadores de daño por estrés oxidativo, no es posible inferir el mecanismo del efecto identificado.

\section{Resumen}

Introducción: Durante la infección de la malaria, tanto el parásito como el hospedero están bajo los efectos de estrés oxidativo, dado que se aumenta la producción de especies reactivas del oxígeno, las cuales pueden inducir daños en el ADN debido a su gran efecto genotóxico. Objetivo: Evaluar el efecto genotóxico en linfocitos humanos en una cohorte de pacientes con malaria de Medellín y Quibdó. Métodos: Se realizó un estudio observacional transversal en 100 personas con malaria y 100 controles sanos. Se incluyeron pacientes infectados con Plasmodium, que consultaron en el Instituto Colombiano de Medicina Tropical de Medellín y el Hospital Ismael Roldán Valencia de Quibdó. Se realizó una valoración transversal del efecto (daño genético) mediante electroforesis en gel de células individuales (ensayo Cometa). Resultados: El promedio de longitud de la cola de los pacientes $(26,9 \pm 9,8)$ fue significativamente mayor que la media de los controles sanos $(14,8 \pm 3,2)(\mathrm{p}<0,01)$. Conclusión: Se evidenció en la población de estudio que la infección por malaria generó genotoxicidad, no así variables como tabaquismo, tratamiento antimalárico y ocupación.

\section{Referencias bibliográficas}

1.- Carmona J. La malaria en Colombia, Antioquia y las zonas de Urabá y Bajo Cauca: panorama para interpretar la falla terapéutica antimalárica. Iatreia 2003; 16 (4): 299-317.

2.- Padilla J C. Tratamiento de la malaria en Colombia. Infectio 1998; 2 (1): 49-56.

3.- Pabón A, Carmona J, Burgos L C, Blair S. Oxidative stress in patients with noncomplicated malaria. Clin Biochem 2003; 36 (1): 71-8.

4.- Melgarejo E. Free radicals in human pathology, its importance and its clinical complications. In: Astract: Congress of Cardiology. Caracas: Venezuelan Society of Cardiology; 1992.

5.- Ray G N, Shahid M, Husain S A. Effect of nitric oxide and malondialdehyde on sister-chromatid exchanges in breast cancer. Br J Biomed Sci 2001; 58 (3):169-76.

6.- Trueba G P. Oxygen free radical and antioxidant defense mechanism in cancer. Front Biosci 2004; 9: 2029-44.

7.- Flora S J. Role of free radicals and antioxidants in health and disease. Cell Mol Biol 2007; 53 (1): 1-2.

8.- Johnston W T, Mutalima N, Sun D, Emmanuel B, Bhatia K, Aka P, et al . Relationship between Plasmodium falciparum malaria prevalence, genetic diversity and endemic Burkitt lymphoma in Malawi. Scientific Reports 4: 3741.

9.- Organización Panamericana de la Salud, Guía para atención clínica integral del paciente con malaria [Internet]. Bogotá: Ministerio de la Protección Social; 2010 [fecha de acceso: 14 de febrero de 2013]. Disponible en: http:/www.paho.org/col/ index.php?option $=$ com_docman\&task $=\mathrm{doc}$ download\&gid=1220\&Itemid=.

10.- Costa P M, Ferreira P M, Bolzani Vda S, Furlan M, de Freitas Formenton Macedo Dos Santos V A, Corsino J, et al. Antiproliferative activity of pristimerin isolated from Maytenus ilicifolia (Celastraceae) in human HL-60 cells. Toxicol in vitro 2008; 22 (4): 854-63.

11.- Singh N P, McCoy M T, Tice R R,
Schneider E L. A simple technique for quantitation of low levels of DNA damage in individual cells. Exp Cell Res 1988; 175 (1): 184-91.

12.- Pandrangi R, Petras M, Ralph S, Vrzoc M. Alkaline single cell gel (comet) assay and genotoxicity monitoring using bullheads and carp. Environ Mol Mutagen 1995; 26 (4): 345-56.

13.- Ejchart A, Sadlej-Sosnowska N. Statistical evaluation and comparison of comet assay results. Mutat Res 2003; 534: 85-92.

14.- Brink A, Schulz B, Kobras K, Lutz W K, Stoppe H. Time-dependent effects of sodium arsenite on DNA breakage and apoptosis observed in the comet assay. Mutat Res 2006; 603 (2): 121-8

15.- Tice R R, Agurell E, Anderson D, Burlinson B, Hartmann A, Kobayashi A, et al. Single cell gel/ Comet assay: Guidelines for in vitro and in vivo genetic toxicology testing. Environ $\mathrm{Mol}$ Mutagen 2000; 35: 206-221.

16.- Trisnawaty A, Vian L, Sabatier R, Cano J P. In vitro detection of indirect-acting genotoxins in 
the comet assay using Hep G2 cells. Mutat Res 2000; 468 (2): 227-34.

17.- Uhl M, Helma C, Knasmüller S. Single cell gel electrophoresis assays with human-derived hepatoma (HepG2) cells. Mutat Res 1999; 17: 441 (2): 215-24.

18.- Fabbri C, de Cássia Mascarenhas-Netto R, Lalwani P, Melo G C, Magalhães B M. Lipid peroxidation and antioxidant enzymes activity in Plasmodium vivax malaria patients evolving with cholestatic jaundice. Malar J 2013; 12 : 315 .

19.- Bilgin R, Yalcin M S, Yucebilgic G, Koltas I S, Yazar S. Oxidative stress in vivax malaria. Korean J Parasitol 2012; 50 (4): 375-7.

20.- Narsaria N, Mohanty C, Das B K, Mishra S P, Prasad R. Oxidative stress in children with severe malaria. J Trop Pediatr 2012; 58 (2):147-50.

21.- Sanni L A, Fu S, Dean R T, Bloomfield G, Stocker R, Chaudhri G, et al. Are reactive oxygen species involved in the pathogenesis of murine cerebral malaria? J Infect Dis 1999; 179 (1): 217-22.

22.- Weitberg A B. Hydroxy- and hydroperoxy6,8,11,14-eicosatetraenoic acids induce sister chromatid exchanges in cultured mammalian cells. Am J Med Sci 1990; 299 (1): 50-3.

23.- Eisenberg W C, Taylor K, Guerrero R R. Cytogenetic effects of singlet oxygen. J Photochem Photobiol B 1992; 16 (3-4): 381-4.

24.- National Research Council. Complex mixtures: method for in vivo toxicity testing. Washington: National Academy Press; 1988.
25.- Carpenter D, Arcaro K, Spink D. Understanding the human health effects of chemical mixtures. Environ Health Perspect 2002;110: 25-37.

26.- Hoffmann H, Hogel J, Speit G. The effect of smoking on DNA effects in the comet assay: a meta-analysis. Mutagenesis 2005; 20 (6): $455-66$.

27.- vom Brocke J, Krais A, Whibley C, Hollstein M C, Heinz H. Schmeiser. The carcinogenic air pollutant 3-nitrobenzanthrone induces GC to TA transversión mutations in human p53 sequences. Mutagenesis. 2009; 24 (1): 17-23.

28.- Teixeira J P, Collins A, Laffon B, Anderson D. Genotoxicology and technical advanced. In: Proceedings: 10th International Comet Assay Workshop. Portugal: ICAW; 2013. 